\title{
Numerical Simulation of Temperature and Thermal Stress Fields in Cast-hot-working-die Steel under High Density Electropulsing
}

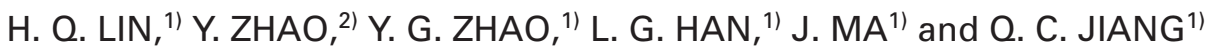 \\ 1) Key Laboratory of Automobile Materials of Ministry of Education and Department of Materials Science \& Engineering, Jilin \\ University, No. 142 Renmin Street, Changchun 130025, P. R. China. E-mail: zhaoyg@mail.jlu.edu.cn \\ 2) School of Communication Engineering, Jilin University, No. 142 Renmin Street, Changchun 130025, P. R. China.
}

(Received on December 3, 2007; accepted on April 21, 2008)

\begin{abstract}
The temperature field and thermal stress field of cast-hot-working-die (CHWD) steel under high density electropulsing were investigated by finite element analysis method. The simulation analysis showed that, during the electropulsing discharging process, the existence of specimen notch resulted in the concentration effect of electric current, namely that the increase of current density in local position. The electric current with high density induced the concentration release of Joule heat and the formation of heat affected zone (HAZ) ahead of specimen notch tip. Because the expanding of HAZ with high temperature was restricted by the neighboring matrix with low temperature, the thermal compressive stress formed in the former during electropulsing discharging. The metallurgy process, under the condition of high temperature and pressure and accompanying thermal compressive stress, was regarded as being beneficial for enhancing mechanical properties and thermal fatigue resistance of CHWD steel and prolonging service lifetime of die in extremely high temperature condition.
\end{abstract}

KEY WORDS: hot working die steel; temperature field; thermal stress field; finite element analysis; electropulsing.

\section{Introduction}

The improvement of thermal fatigue performance of metal materials by electropulsing stimulation in the intermediate stage of thermal fatigue cycle has been repeatedly reported in many papers recently. ${ }^{1-3)}$ During electropulsing discharging process, the electric current lines surpass and concentrate around specimen notch tip and the circle or elliptical heat affected zone (HAZ) comes forth due to the increased Joule heat liberation. $^{2-4)}$ The result of thermal fatigue test showed that the resistance to thermal fatigue was much improved in the HAZ after the electropulsing stimulation. ${ }^{2,4)}$ Because the discharging duration is less than a quarter of one second, some instantaneous experimental data, such as temperature field, stress field, maximum temperature rise, stress state and maximum stress, are not directly measured at elevated temperature. Therefore, it is necessary that the numerical simulation is carried out to study temperature field and accompanying thermal stress field for further illuminating the acting mechanism of electropulsing in respect of forming the HAZ and improving the thermal fatigue resistance.

The finite element analysis is an effective means for the analysis of planar or three-dimensional temperature and stress fields. ${ }^{5-7)}$ In the present study, the temperature fields of CHWD steel under the action of electropulsing with different current densities were simulated by means of finite element analysis and the numerical results were calculated by indirect coupling structural analysis to analyze the ther- mal stress distribution on the surface of electrostimulated specimen. The simulation results are beneficial for investigating the mechanism of the HAZ formation and the enhanced thermal fatigue resistance.

\section{Analytical Conditions}

\subsection{Investigated Object}

The investigated object in the present study is a thermal fatigue specimen $\left(40 \times 12 \times 3 \mathrm{~mm}^{3}\right.$, as shown in Fig. 1) selected from CHWD steel. An additional notch with gage width of $0.18 \mathrm{~mm}$ and length of $3 \mathrm{~mm}$ was machined at the central section the longitudinal direction for the investigation of thermal fatigue crack. During the electropulsing discharging process, the specimen was directly connected in the discharging circuit at the ambient temperature. The other physical parameters of the investigated object required for simulation calculation are listed in Table $\mathbf{1 .}$

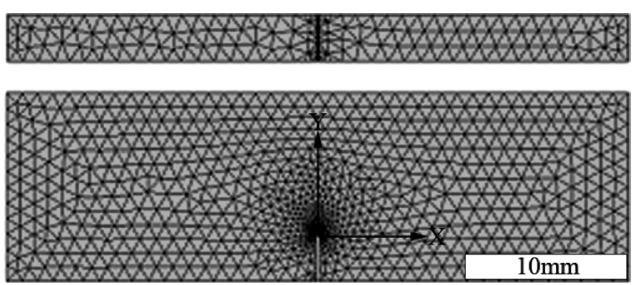

Fig. 1. Elements for meshed model for the thermal fatigue specimen. 
Table 1. Physical parameters of CHWD steel for numerical calculation.

\begin{tabular}{cc}
\hline Physical properties items & Values or fitting expression \\
\hline Density, $10^{3} \mathrm{~kg} / \mathrm{m}^{-3}$ & $7.76-0.003 \mathrm{~T}$ \\
Young's modulus, $\mathrm{GPa}$ & $207-0.12 \mathrm{~T}$ \\
Poisson ratio & 0.3 \\
Specific heat capacity, $\mathrm{J} /\left(\mathrm{kg} \cdot{ }^{\circ} \mathrm{C}\right)$ & $440-0.01 \mathrm{~T}+7.30 \mathrm{~T}^{2}$ \\
Heat conduction coefficient, $\mathrm{W} /\left(\mathrm{m}^{2} \cdot{ }^{\circ} \mathrm{C}\right)$ & $18.5+0.01 \mathrm{~T}$ \\
Heat expanding coefficient, $10^{-6} /{ }^{\circ} \mathrm{C}$ & $10.35+0.003 \mathrm{~T}$ \\
Electric resistivity, $10^{-6} / \Omega \cdot \mathrm{m}$ & $0.44+0.04 \mathrm{~T}$ \\
Convection film coefficient, $\mathrm{W} /\left(\mathrm{m}^{2} \cdot{ }^{\circ} \mathrm{C}\right)$ & 12 \\
\hline
\end{tabular}

Note: $\mathrm{T}$ is instantaneous heating temperature induced by electropulsing sitimulation.

\subsection{Establishment and Meshing of Model}

The investigated model was established by using threedimensional SOLID69 element. Initially, a rectangle was built in the two-dimensional plane, from which a notch was subtracted at the central section in width direction. Then the rectangle with a notch was moved along the axis perpendicular to the plane. Therefore, a SOLID69 was formed for numerical simulation. The geometry and meshing of the SOLID69 are shown in Fig. 1. After thermal analysis, the SOLID69 thermal element was changed to the structural element SOLID45 to analyze the thermal stress.

\subsection{Initial and Boundary Conditions}

It is assumed that the initial temperature of specimen is room temperature $20^{\circ} \mathrm{C}$. In the present thermal analysis simulation, the circumstance was not adiabatic and the surface heat convection film coefficient was set as $12 \mathrm{~W} /\left(\mathrm{m}^{2} \cdot \mathrm{K}\right)$. The thermal stress value is regarded as $0 \mathrm{MPa}$ at the reference temperature of $20^{\circ} \mathrm{C}$. Because the discharging duration was relatively short, the surfaces of electrostimulated specimen were regarded as being absent of effective opportunity for expanding to their normal directions. Therefore, the surfaces of investigated model were partially restricted for the ideal thermal stress analysis.

\subsection{Calculation for the Field Distributions of Temper- ature and Thermal Stress}

The ANSYS software was used in the present simulation to build the SOLID69 model for the thermal analysis and the SOLID45 model for structural analysis, and to mesh the SOLID69 and SOLID45 models, respectively. The initial and boundary conditions as listed in Table 1 were defined by inputting physical parameters of thermal and structural properties in the dialog boxes of ANSYS software. After imposing the determined electric field on the two ends of the founded model, the ANSYS program was executed for the calculation of temperature field by nonlinear solution for 20 equilibrium iterance. The general postprocessing was performed for outputting contour plots of temperature and thermal stress fields. In addition, the linear distributions of temperature and thermal stress along the $X$ and $Y$ coordinates in Fig. 1 are also solved in the postprocessing, respectively.

\section{Analytical Results and Discussion}

\subsection{Temperature Distribution around the Notch}

Figure 2 shows contour bands for temperature fields of CHWD steels electrostimulated with different current densities: (a) 70, (b) 80 , (c) 90 and (d) $100 \mathrm{MA} / \mathrm{m}^{2}$ instantly at the end of discharging. It is easily found in Fig. 2 that the concentrated heat release occurred ahead of the notch tip and was characterized by the high temperature contour band. During electropulsing discharging process, the electric field lines surpass the notch tip deviously and the electric current density is exceedingly high ahead of the notch tip, which results in concentrated heat release under the function of Joule heat effect. In the practical experiment, ${ }^{2,4)}$ once the temperature of CHWD steel surpasses tempering temperature $600^{\circ} \mathrm{C}$, large amount of nanosized carbides separate dispersively from martensite matrix, which enhances the resistance against acid liquid corrosion. Therefore, the region with heating temperature above $600^{\circ} \mathrm{C}$ is different from steel matrix and named as HAZ. It is observed from Fig. 2 that the HAZ is distributing mainly ahead of the notch tip. The further observation by comparing Figs. 2(a)-2(d), shows that the acreage of HAZ enlarges with the increase of electric energy inputting into specimen by elevating current density from Fig. 2(a) $70 \mathrm{MA} / \mathrm{m}^{2}$ to Fig. 2(b) 80,2 (c) 90 and 2(d) $100 \mathrm{MA} / \mathrm{m}^{2}$. The temperature variation on the HAZs on CHWD steels with the increase of discharging time during electropulsing stimulation with different current densities is shown in Fig. 3. The temperature rise of electrostimulated specimen induced by motion of electrons can be given in the following expression:

$$
\Delta T=\rho j^{2} t\left(c_{\mathrm{p}} d\right)^{-1}
$$

where $j$ is the current density of electropulsing, $t$ is the discharging duration, and $\rho, c_{\mathrm{p}}$ and $d$ are the electrical resistivity, the specific heat and the density of specimen, respectively. The qualitative theoretical analysis from the abovementioned expression showed that the specimen temperature rise $\Delta T$ is proportional to the second power of current density $j$ and discharging duration $t$ of electropulsing, respectively. Consequently, the specimen temperature rise $\Delta T$ increased in the nonlinear way with the increase of discharging duration $t$ of electropulsing. What's more, it is also observed from Fig. 3 that the elevation of current density of electropulsing is favorable for the increase of instantaneous temperature rise on the surface of electrostimulated specimen. For example, at the end of electropulsing discharge, 

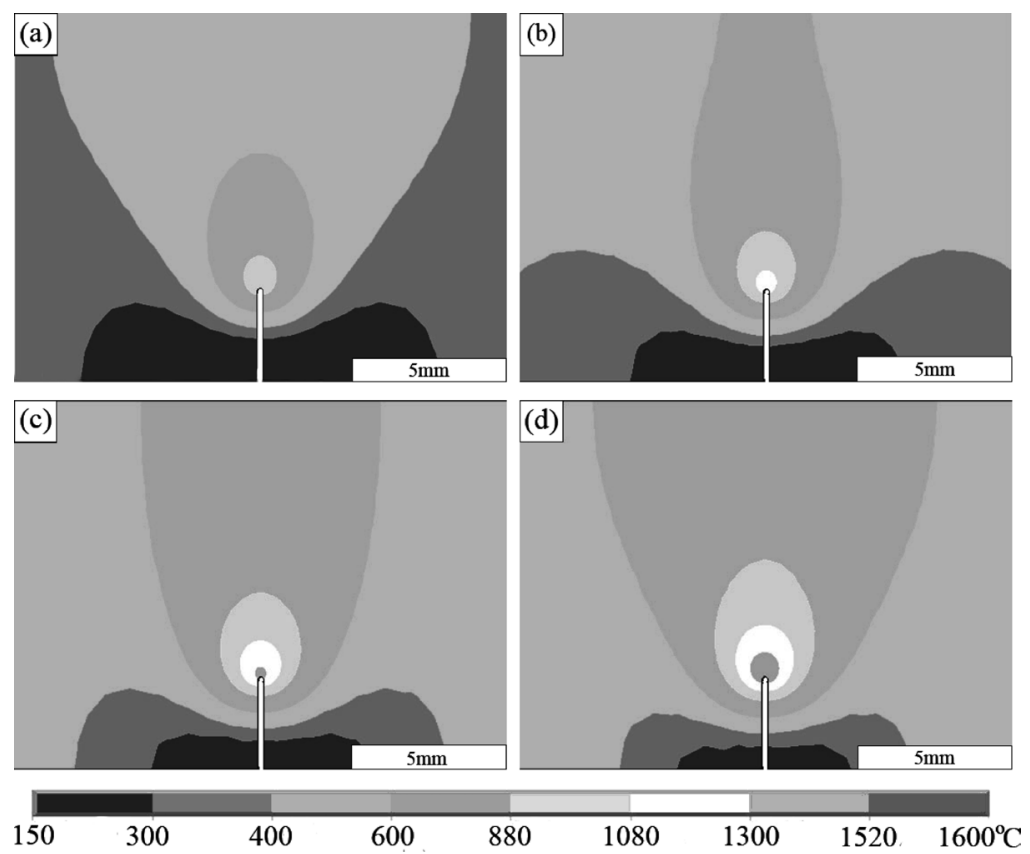

Fig. 2. Contour bands for temperature fields of CHWD steels instantly at the end of discharging with different current densities: (a) 70, (b) 80, (c) 90 and (d) $100 \mathrm{MA} / \mathrm{m}^{2}$.

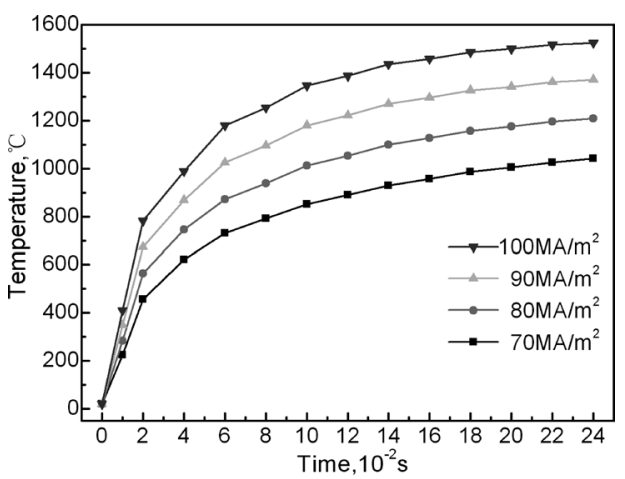

Fig. 3. Maximum temperature variation versus discharging time on the HAZs on CHWD steels electrostimulated with different current densities: 70, 80, 90 and $100 \mathrm{MA} / \mathrm{m}^{2}$.

the instantaneous maximum temperature rises of electrostimulated specimens are $1042,1209,1370$ and $1520^{\circ} \mathrm{C}$ under the action of electropulsing for $240 \mathrm{~ms}$ with different current densities: $70,80,90$ and $100 \mathrm{MA} / \mathrm{m}^{2}$, respectively. With the further slight increase of current density above $100 \mathrm{MA} / \mathrm{m}^{2}$, the partial surface on electrostimulated specimen will exceed the melting point $\left(1520^{\circ} \mathrm{C}\right)$ of CHWD steel material. The melting damage will occur ahead of the notch and destroy the surface quality of die material, so the peak value of current density is regarded as $100 \mathrm{MA} / \mathrm{m}^{2}$.

The investigation of the temperature distribution, such as the HAZ range on the electrostimulated specimen, provides a basis for the further experimental research about the effect of electropulsing stimulation on the microstructure and thermal fatigue behavior of CHWD steel. Only knowing the HAZ range, the microstructure of HAZ could be studied and distinguished from that of the matrix. Figure 4 showed the temperature distribution around specimen notch tip in different directions: (a) $X$-coordinate and (b) $Y$-coordinate (the coordinate as depicted in Fig. 1) instantly at the end of discharging with different current densities. It proved that the regions with high temperature are mainly distributed around the specimen notch tip in Fig. 4 from the viewpoint of numerical simulation. It can be observed from Figs. 4(a) and $4(\mathrm{~b})$ that the HAZ distributes mainly within the range of $\pm 4 \mathrm{~mm}$ along the $X$-coordinate and $6 \mathrm{~mm}$ along the $Y$ coordinate. In addition, the temperature of the region beside the notch bottom is relatively lower than that of the other matrix due to the passage of electric current with lower current density.

\subsection{Thermal Stress Distribution around the Notch}

The variation of temperature around the notch tip when electric current is passing through the specimen has been successfully revealed in the above-mentioned section. It has been found from the stimulation result that the temperature of HAZ is obviously higher than that of matrix. Because the expanding of HAZ with high temperature was restricted by the neighboring matrix with low temperature, the thermal compressive stress formed in the former during electropulsing discharging process. The thermal stresses resulting from such uneven temperature distribution are solved and presented in the present section. Figure 5 shows contour bands for thermal stress fields of CHWD steels electrostimulated with different current densities: (a) 70, (b) 80, (c) 90 and (d) $100 \mathrm{MA} / \mathrm{m}^{2}$ instantly at the end of discharging. It is observed that the thermal stress is mainly distributing around the specimen notch tip. The transient maximum thermal stresses increase from $1140 \mathrm{MPa}$ to 1190,1260 and $1330 \mathrm{MPa}$ for the electropulsing stimulation with current densities of $70,80,90$ and $100 \mathrm{MA} / \mathrm{m}^{2}$, respectively. The acreage of equal stress contour band also increases with the elevating of current density. The distribution of thermal stress on the surface of electrostimulated specimen along different directions: (a) $X$-coordinate and (b) $Y$-coordinate are schematically shown in Fig. 6. The thermal stress distributes mainly within the range of $\pm 4 \mathrm{~mm}$ along the $X$ coordinate and $6 \mathrm{~mm}$ along the $Y$-coordinate, which is con- 

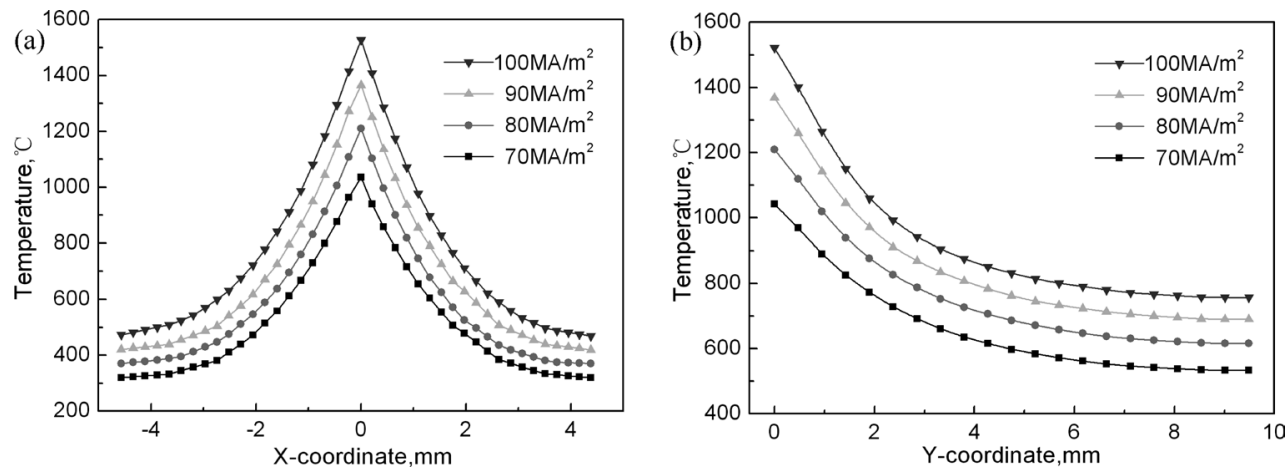

Fig. 4. Temperature distribution in different directions: (a) $X$-coordinate and (b) $Y$-coordinate around specimen notch instantly at the end of discharging with different current densities: 70, 80,90 and $100 \mathrm{MA} / \mathrm{m}^{2}$.
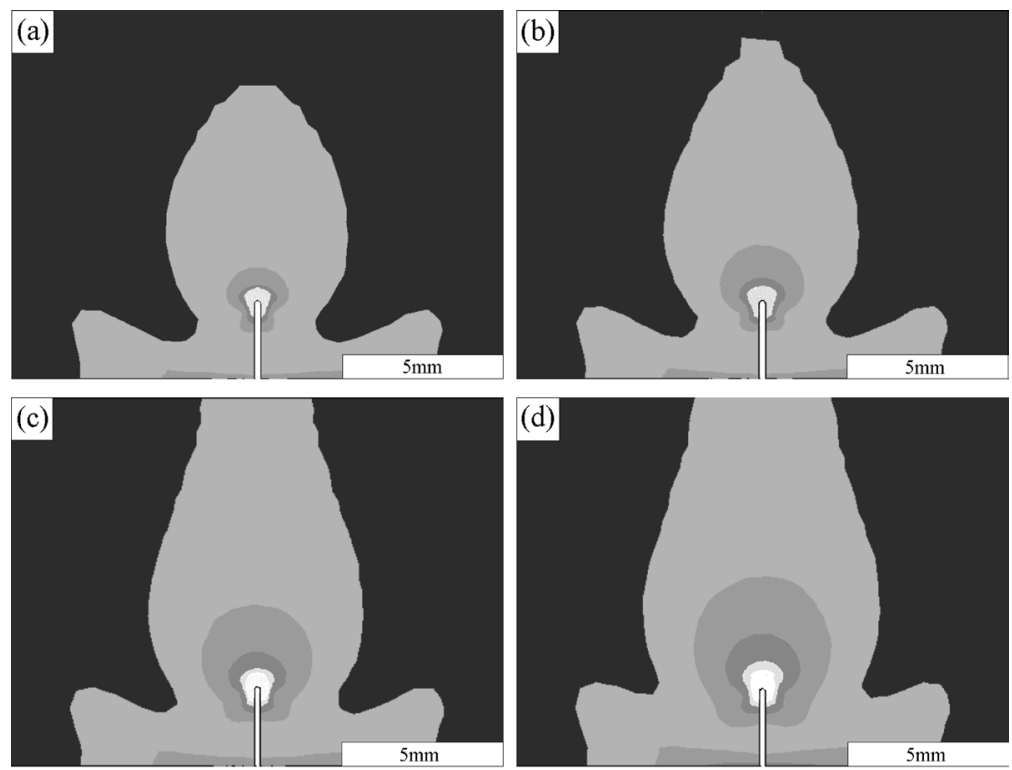

100

750

$\begin{array}{llll}1000 & 1250 & 1500 & 2000 \mathrm{MPa}\end{array}$

Fig. 5. Contour bands for Von Mises Stress on the surface of CHWD steel instantly at the end of discharging with different current densities: (a) 70, (b) 80 , (c) 90 and (d) $100 \mathrm{MA} / \mathrm{m}^{2}$.
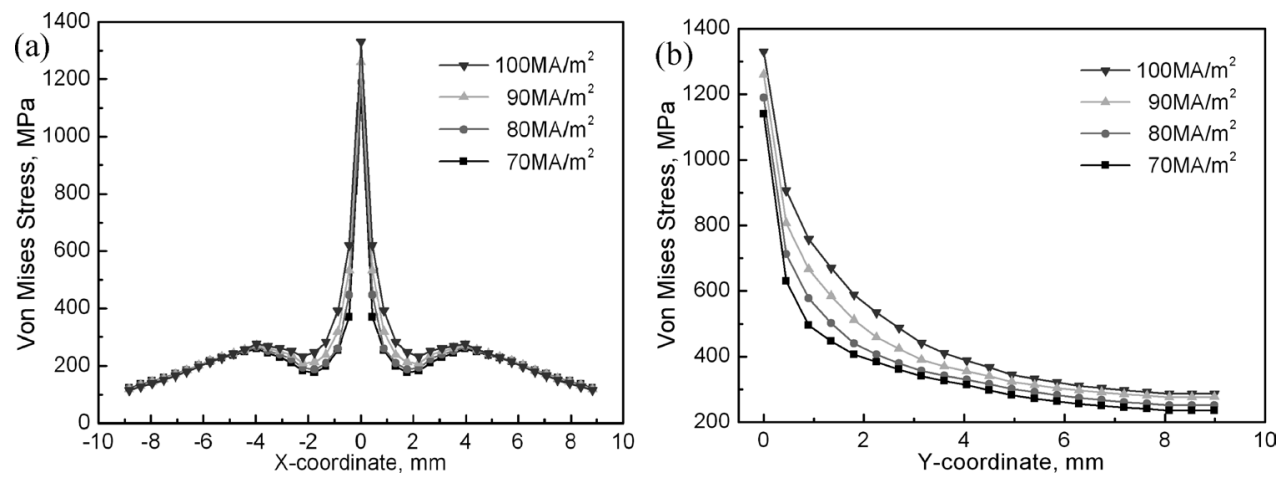

Fig. 6. Stress distribution along different directions: (a) $X$-coordinate and (b) $Y$-coordinate on the HAZ surface of CHWD steel stimulated by electropulsing with different current densities: 70, 80, 90 and $100 \mathrm{MA} / \mathrm{m}^{2}$.

sistent with the analysis result of temperature distribution. In addition, the stress of the region beside notch bottom is relatively higher than that of the other matrix, although the temperature of corresponding region is much lower than that of other matrix and HAZs. The reason is regarded as the temperature gradient present between the region near the notch bottom and that far from it. It is necessary to point out that the thermal stress in the present paper is Von
Mises stress. Plastic deformation will initiate when Von Mises stress reaches a certain level. After pulse electric current discharging, the cooling process occurs and the residual compressive stress will form around the specimen notch. The X-ray diffraction analysis shows that the average value of residual compressive stress on the surface ahead of notch tip of electrostimulated specimens increased from $744 \mathrm{MPa}$ to 869,996 and $1164 \mathrm{MPa}$ with the elevation of 
current density form $70 \mathrm{MA} / \mathrm{m}^{2}$ to 80,90 and $100 \mathrm{MA} / \mathrm{m}^{2}$, respectively. The compressive stress forming by electrostimulation will reduce the peak value of tensile stress forming during the heating process of thermal fatigue test and is beneficial for increasing the resistance against thermal crack initiation and propagation of die material. Therefore, high density electrostimulation is favorable for prolonging the service lifetime of hot working die.

\section{Conclusions}

(1) The temperature field can be simulated by finite element method. The circle/elliptic HAZ mainly forms and distributes around the specimen notch tip during electropulsing stimulation process. The acreage of HAZ increases with the increase in current density of electropulsing.

(2) The thermal stress field on the specimen could be calculated by indirect coupling structural analysis after thermal analysis. The electropulsing stimulation induced the formation of thermal compressive stress on the specimen surface. The elevation of current density is beneficial for increasing compressive stresses on the surface of electrostimulated specimens.

(3) The simulation analysis of temperature field and thermal stress field provide theoretical basis for the experimental research on the enhancement of thermal fatigue resistance by electropulsing stimulation.

\section{Acknowledgements}

This work is supported by the Research Fund for Doctoral Program of Higher Education (No. 20050183053), the 863 Program (No. 2006AA03Z566) granted by the Ministry of Science and Technology of the People's Republic of China and the Project 985-Automotive Engineering of Jilin University.

\section{REFERENCES}

1) V. V. Levitin and S. V. Loskutov: Solid State Commun., 131 (2004), 181.

2) Y. G. Zhao, Y. H. Liang, W. Zhou, Q. D. Qin and Q. C. Jiang: ISIJ Int., 45 (2005), 410.

3) L. B. Zuev, O. V. Sosnin, S. F. Podboronnikov, V. E. Gromov and S. N. Gorlova: Solids, 45 (2000), No. 3, 309.

4) H. Q. Lin, Y. G. Zhao, Z. M. Gao and L. G. Han: Mater. Sci. Eng. A, (2008), 93

5) G. X. Cai and F. G. Yuan: Adv. Eng. Software, 29 (1998), No. 3-6, 297.

6) G. X. Cai and F. G. Yuan: Int. J. Fract., 96 (1999), 279.

7) C. S. Li, X. H. Liu and J. Z. Xu: J. Mater. Eng. Perform., 11 (2002), No. $2,215$. 